\title{
O TREINAMENTO MENTAL MELHORA O DESEMPENHO DO FUNDAMENTO RECEPÇÃO DE ATLETAS FEMININAS DE VOLEIBOL
}

\author{
Fernando Souza Manhães ${ }^{1}$, José Carlos Gomes dos Santos Jr ${ }^{1}$, Carlos Eduardo dos \\ Santos Peixoto ${ }^{2}$, Nilo Terra Arêas Neto ${ }^{2} \&$ Mauricio Rocha Calomeni ${ }^{2 *}$
}

\begin{abstract}
RESUMO
MANHÃES, F.S.; JUNIOR, J.C.G.S.; PEIXOTO, C.E.S.; NETO, N.T.A. \& CALOMENI, M.R. O treinamento mental melhora o desempenho do fundamento recepção de atletas femininas de voleibol. Perspectivas Online: Biológicas \& Saúde. v. 9, n 30, p.1-12, 2019.
\end{abstract}

Este estudo objetivou investigar como uma intervenção com treinamento mental (imaginação) afetaria o desempenho de jogadoras de voleibol amadoras em relação ao fundamento recepção de manchete. A investigação seguiu o delineamento quaseexperimental com pré e pós-teste e um grupo controle. Os dados foram coletados de uma amostra de 20 atletas, jogadoras de vôlei, do sexo feminino, com idade entre 13 a 17 anos. A amostra foi dividida em dois grupos: Grupo Controle (GC), com 10 sujeitos que participaram apenas do teste de capacidade imaginativa e do escore de rendimento, e
Grupo Experimental (GE), composto por 10 sujeitos, os quais participaram além dos mesmos testes do GC também do protocolo de treinamento mental. Para analisar os dados foi usado o teste de normalidade, Teste $\mathrm{T}$ pareado e não pareado. Os resultados demonstraram melhora estatisticamente significante do grupo experimental no desempenho da recepção na posição 6 após a intervenção do treinamento mental. Concluise que, o treinamento mental é um método eficiente para melhoria do desempenho na recepção do voleibol, e deve ser aplicada de maneira específica para cada situação.

Palavras-chave: Treinamento mental; Aprendizagem motora; Desempenho Esportivo. 


\begin{abstract}
This study aimed to verify as an intervention with mental training (imagery) would affect the performance of amateur volleyball players in relation to headline reception foundation. The investigation followed the quasiexperimental design with pre and post tests and one control group. The data were collected of a sample of 20 athletes, volleyball player, of female gender, with age between 13 and 17 years. The sample was divided in two groups: Control Group (GC), with 10 individuals which only participated of the tests of ability imaginative and

performance score, and Experimental Group (GE), formed by 10 individuals, that beside the same tests applied to GC they participated also of mental training protocol. To analyses the data was used the normality test, paired and non-paired $\mathrm{T}$ test. The results showed statistically relevant increase of experimental group in the performance of reception in the 6 position after a mental training intervention. It is concluded that the mental training is an effective method to increase the performance in the volleyball reception and should be applied in a specific way for each situation.
\end{abstract}

Keywords: Mental training; Motor learning, Sportive performance.

\footnotetext{
${ }^{1}$ Institutos Superiores de Ensino do CENSA -ISECENSA - Profissional da Educação Física - Rua Salvador Correa, 139, Centro, Campos dos Goytacazes, RJ, CEP: 28035-310, Brasil.

2 Pesquisador do Laboratório de Biociências da Motricidade Humana- LABIMH/ ISECENSA

(*)e-mail: mauriciocalomeni@gmail.com

Data de recebimento: 18/03/2019 Aceito para publicação: 11/06/2019
} 


\section{PERSPECTIVASonline CIÊNCIAS BIOLÓGICAS E da SaÚde}

\section{INTRODUÇÃO}

Durante sua trajetória, o voleibol teve grandes mudanças em seu regulamento e principalmente na forma em que os atletas praticam este esporte. Para acompanhar esse desenvolvimento, os atletas se condicionam fisicamente para executar as demandas do esporte que são cada vez maiores. Sendo assim, a cada década as equipes começaram a buscar formas de melhor preparar seus atletas para os jogos, nesse sentido a ciência vem apontando o treinamento mental como efetivo sobre os aspectos cognitivos relacionado ao desempenho (FILGUEIRAS et al, 2012).

O treinamento mental pauta-se no fato de que ao se pensar em um ato motor já é capaz de melhorar a transmissão sináptica, melhorando a capacidade de execução de determinados movimentos, uma vez que a simulação mental de um determinado movimento emprega os mesmos mecanismos neurais utilizados na sua execução (RODRIGUES et al, 2003). Dessa forma, o treinamento mental vem sendo verificado em diversos contextos esportivos, tais como a natação (GOMES, MARINHO e BENDA, 2016; FORTES et al, 2017), arremesso de peso (REZENDE, SILVA e SOUZA, 2016), e, treinamento de força (ORNELAS et al, 2017).

Especificamente no voleibol, em estudo recente conduzido por Branco et al, (2017) com atletas amadoras com idades entre 13 e 17 anos, mostrou que o estímulo do treinamento mental acarretou um aumento na atividade eletromiográfica na musculatura dos membros inferiores durante a simulação mental do fundamento bloqueio. O que corroborou a afirmação de Castro e Santos (2007) que quando uma pessoa pensa sobre um ato ou movimento de alguma parte do corpo, ocorre um aumento nos registros eletromiográficos na musculatura da área específica.

Dentre os diversos fundamentos do voleibol a recepção se destaca por ser o momento em que a equipe inicia a organização do ataque, estabelecendo uma ação comunicativa com o levantamento (FAGUNDES et al, 2017). Além disso, Ribas et al (2014) apontam que para uma equipe ser bem-sucedida nos seus objetivos "as ações de cooperação deverão ser altamente organizadas". Desta forma, o objetivo do estudo foi testar a hipótese de que a utilização de um protocolo de treinamento mental produziria alterações significativas na eficiência do fundamento de recepção em atletas amadoras de voleibol.

\section{MATERIAIS E MÉTODOS}

Esse estudo foi aprovado com número do parecer consubstanciado 2.779 .744 emitido pelo do Comitê de Ética em Pesquisa com Seres Humanos do ISECENSA.

A amostra da pesquisa foi formada por atletas amadoras de voleibol do sexo feminino inseridas nas equipes do ano de 2018 de duas instituições privadas do município de Campos dos Goytacazes com tradição na participação de jogos escolares de nível regional e estadual, com faixa etária de 13 a 17 anos de idade. Sendo assim, foram incluídas na pesquisa atletas do sexo feminino que jogam voleibol a pelo menos 1 ano, que não possuam qualquer tipo de deficiência auditiva e que frequentem os treinos de seus respectivos times regularmente. Foram excluídas atletas que os responsáveis não concordaram com a participação no estudo, que não concordavam voluntariamente com a participação, que estivessem com qualquer tipo de lesão no período da pesquisa, e que, ficaram abaixo do ponto de corte de $40 \%$ de acertos no teste de capacidade 
imaginativa que foi determinado especificamente para esse estudo como forma de se garantir um status mínimo de capacidade imaginativa.

Dessa forma, para atender o critério de exclusão foi utilizado o Questionário de Imaginação Motora (MIQ-R) que já foi utilizado em trabalhos com desportistas brasileiros (ALMEIDA et al, 2008; MENDES, 2012). O questionário é estruturado por meio de 8 (oito) sugestões de imagética visual e imagética cinestésica em concomitância com uma escala de percepção por parte do avaliado do grau de dificuldade encontrado na realização da sugestão feita, sendo ora pedido para que o indivíduo se visualize executando um determinado movimento, e em seguida se sinta executando o mesmo.

Por não se ter encontrado na literatura pesquisa protocolos validados que atendessem ao objetivo da pesquisa, foi criado um instrumento específico para avaliação da efetividade das atletas no fundamento recepção. Esse instrumento se tratou de um escore de acertos no fundamento recepção, caracterizado pela quantidade de recepções corretas a partir da posição 6 da quadra de voleibol. Optou-se especificamente por essa posição para que houvesse uma padronização no protocolo e por esse ser uma posição central na quadra de voleibol, o que facilita o direcionamento do saque. Para tanto, foram realizados vinte saques utilizando a técnica do "saque por baixo", efetuados pelo técnico e/ou assistente de voleibol de forma que fossem homogêneas a precisão e potência dos saques. Foram considerados válidos os saques que não exigiram deslocamento maior do que dois passos da passadora. Por sua vez, foi determinado como recepção correta quando essa não exigir um deslocamento maior de dois passos da levantadora que se encontrava na posição 3. A opção por dois passos para validação tanto do saque quanto da recepção foi feita com os objetivos de se padronizar o protocolo proposto e para se garantir um nível mínimo de precisão nos dois fundamentos. Foi considerado para determinar o rendimento no fundamento recepção, o percentual de acertos de cada atleta utilizando-se como base os critérios descritos acima.

Por fim, o último instrumento foi o protocolo de treinamento mental que consistiu em sugestões imaginativas gravadas em áudio através do programa de edição de áudio Audacity. Assim como no instrumento anteriormente descrito, por não se ter encontrado na literatura pesquisada protocolos que atendessem ao objetivo da pesquisa, optou-se por propor um protocolo de treinamento mental específico no qual inicialmente foi pedido às atletas que fechassem os olhos e se imaginassem executando os movimentos da recepção, dando ênfase inicialmente no passo a passo da técnica, como posição de mão, posição do braço, detalhes do fundamento da recepção como manter os cotovelos estendidos, flexão de joelhos, imaginar uma pessoa do outro lado da quadra sacando uma bola, imaginar o saque vindo na sua direção, imaginar o deslocamento para receber o saque, sugerir que imaginem a bola tocando no antebraço, imaginar efetuando o passe para a levantadora, pedir para que a atleta se imagine em uma situação de jogo com torcida, realizando o fundamento recepção perfeitamente. Todo protocolo de treinamento mental teve tempo total de 15 minutos, sendo os primeiros 5 minutos destinados ao aumento da concentração e internalização dos pensamentos. Em seguida, nos próximos 5 minutos foi solicitado que os indivíduos se sentissem como se estivessem realizando o movimento da manchete passo a passo. Por fim, nos últimos 5 minutos, os indivíduos foram induzidos a se imaginar realizando recepções em diferentes situações específicas do voleibol, seja de saque, ataque, em ginásios lotados, jogos importantes. Foi decidido gravar o áudio da voz com os comandos para o treinamento mental, para se evitar variações na forma em que os comandos eram passados para atletas, e assim se garantir uma padronização entre as sessões de treinamento mental. 
Para utilização dos instrumentos descritos, foi contatado inicialmente os técnicos de ambas instituições para selecionar as atletas que atendessem aos critérios de inclusão determinados para o estudo. Foi explicado para as atletas o objetivo do estudo e foi solicitado a assinatura do Termo de Consentimento Livre e Esclarecido (TCLE) pelos responsáveis, e do Termo de Assentimento pelas próprias atletas.

O primeiro teste que foi aplicado em todas as participantes do estudo com objetivo de se determinar a capacidade imaginativa das atletas foi o MIQ-R. Através deste foi possível garantir que todas as participantes tivessem capacidade imaginativa suficiente para participar do estudo. Tal questionário foi aplicado individualmente em sala cedida pelas próprias escolas em que os times treinavam, e previamente preparada para minimizar agentes dispersores. E, nessa avaliação verificou-se que todas as atletas alcançaram pontuação acima da linha de corte especificada para a pesquisa.

O segundo procedimento também foi feito antes da intervenção, e com todas as participantes do estudo, tratou-se da determinação do escore de rendimento das atletas no fundamento recepção. Esse escore também foi feito individualmente, na própria quadra onde as atletas estão habituadas a treinar e seguindo o protocolo descrito nos parágrafos acima.

Findada a etapa das avaliações iniciais, foram formados os grupos controle e experimental. Por conveniência, determinou-se que as atletas de uma equipe formariam o grupo controle, e as da outra formariam o grupo experimental. Dessa forma, houve apenas um sorteio simples para se determinar qual equipe seria controle, e qual seria experimental. Foi solicitado ao grupo controle que o técnico mantivesse o planejamento de treinamento previsto para as atletas. Todavia, as atletas inseridas no grupo experimental passaram pelo protocolo de treinamento mental, que também foi aplicado de forma individual. Para tanto antes de cada sessão de treinamento convencional, cada atleta era conduzida a um local em que pudesse se sentar o mais confortavelmente possível para o início do protocolo de treinamento mental.

As sessões de treinamento mental aconteciam 3 vezes por semana, e sempre foram acompanhadas por dois pesquisadores e pelo professor responsável pelo treinamento das atletas. Importante ressaltar que foi solicitado para ambos os treinadores e atletas das equipes que não aplicassem atividades específicas para o fundamento da recepção durante o período da pesquisa, que teve duração de 4 semanas, para não influenciar os resultados. Após o período de intervenção da pesquisa, foi refeito o protocolo de avaliação do rendimento obedecendo os mesmos procedimentos adotados nas avaliações iniciais.

Os dados foram analisados descritiva e inferencialmente. Devido a característica não paramétrica, foram determinados para análise descritiva a mediana, os extremos e o desvio padrão dos escores do percentual de acertos no fundamento recepção nos grupos controle e experimental. Após essa etapa, para análise inferencial aplicou-se testes T pareado para comparação intra grupo, e, não pareado para as comparações intergrupos. Em todas as análises foi determinado o índice de confiança de $95 \%$, ou seja, $\mathrm{p}<0.05$. 


\section{RESULTADOS E DISCUSSÃO}

Os resultados dos testes aplicados aos participantes da pesquisa foram analisados a partir da normalidade, seguidas pelas análises descritivas e inferenciais necessárias para se testar a hipótese de que o treinamento mental seria efetivo para melhoria do fundamento avaliado. A partir da tabela 1 é possível se analisar o comportamento dos dados em cada ensaio experimental.

Tabela 1. Teste de normalidade: Grupo controle x Grupo experimental

\begin{tabular}{|c|c|c|c|c|c|c|c|c|}
\hline & \multicolumn{4}{|c|}{ Grupo Controle } & \multicolumn{4}{|c|}{ Grupo Experimental } \\
\hline & \multirow{2}{*}{$\begin{array}{l}\text { Imagética } \\
\text { Cinestésica } \\
\text { (IC) }\end{array}$} & \multirow{2}{*}{$\begin{array}{c}\text { Imagética } \\
\text { Visual (IV) }\end{array}$} & \multicolumn{2}{|c|}{ Escore de Desempenho } & \multirow{2}{*}{$\begin{array}{l}\text { Imagética } \\
\text { Cinestésica } \\
\text { (IC) }\end{array}$} & \multirow{2}{*}{$\begin{array}{c}\text { Imagética } \\
\text { Visual (IV) }\end{array}$} & \multicolumn{2}{|c|}{ Escore de Desempenho } \\
\hline & & & $\begin{array}{c}\text { Pré- } \\
\text { intervenção }\end{array}$ & $\begin{array}{c}\text { Pós- } \\
\text { Intervenção }\end{array}$ & & & $\begin{array}{c}\text { Pré- } \\
\text { intervenção }\end{array}$ & $\begin{array}{c}\text { Pós- } \\
\text { Intervenção }\end{array}$ \\
\hline $\mathrm{P}$ valor & 0.2113 & 0.0135 & 0.1727 & 0.6394 & 0.2931 & 0.6945 & 0.8855 & 0.3310 \\
\hline $\begin{array}{c}\text { Passou no teste } \\
\text { de Normalidade } \\
(\text { alpha }=0.05) ?\end{array}$ & Sim & Não & Sim & Sim & Sim & Sim & Sim & Sim \\
\hline P valor sumário & $\mathrm{ns}$ & $*$ & $\mathrm{~ns}$ & $\mathrm{~ns}$ & $\mathrm{~ns}$ & $\mathrm{~ns}$ & ns & ns \\
\hline
\end{tabular}

A partir da tabela 1 é possível se afirmar que com exceção dos dados do escore da imagética visual no grupo controle, em todas as outras avaliações os dados apresentaram um comportamento normal. Sendo assim, podendo ser classificados como paramétricos, e credenciando a média aritmética como medida de tendência central. Para os dados da imagética visual no grupo controle, devido à característica não paramétrica, foi adotada a mediana com medida de tendência central.

Determinada a normalidade dos dados serão apresentados os dados estatísticos necessários para análise descritiva. Dessa forma, para facilitar a apresentação e entendimento desses dados, serão apresentados na figura 1 primeiramente os escores da capacidade imaginativa oriundos do teste MIQ-R. 


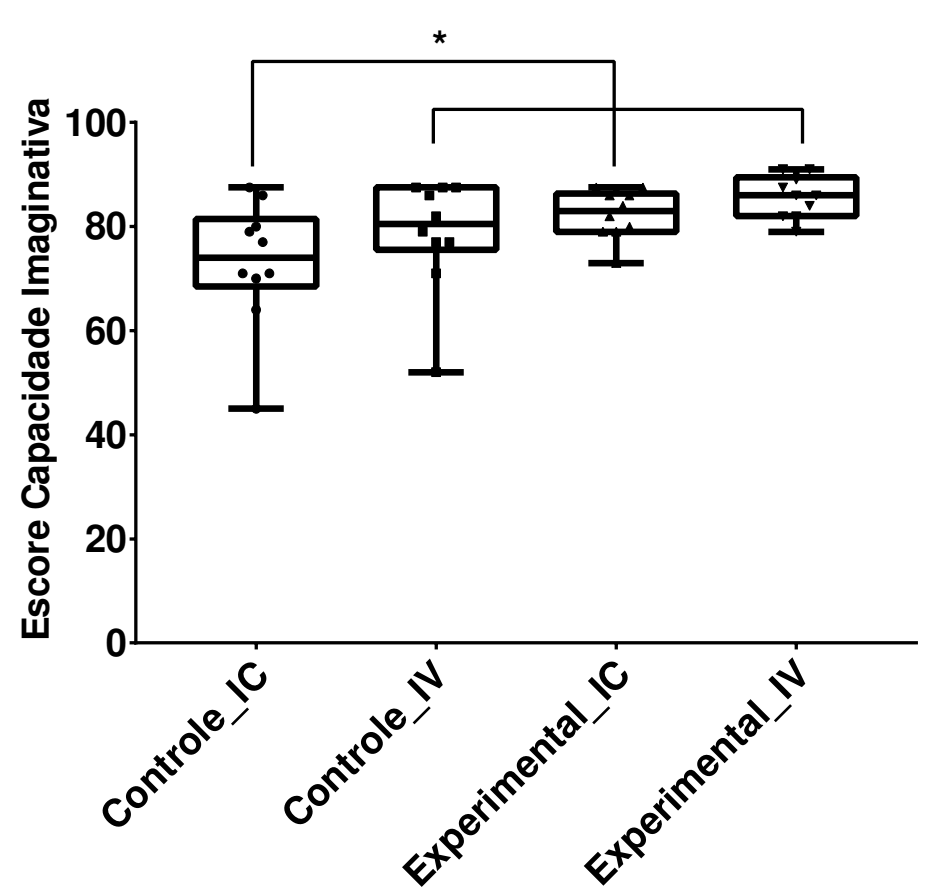

Figura 1. Figura ilustrativa das comparações intergrupos dos escores da capacidade Imaginativa: IC $\mathrm{x} I V . *$ representa diferenças estatisticamente significativas.

A figura 1 mostram que os grupos apresentaram uma diferença estatística em relação a imagética cinestésica, ou seja, as atletas do grupo controle tinham uma capacidade inferior de se sentir realizando os movimentos mentalizados. Em contrapartida na imagética visual os grupos se mostraram estatisticamente iguais, em outras palavras, ambos os grupos mostraram ter facilidade em se visualizar enquanto mentalizavam o movimento proposto. Viana (1996) relata que existem diversos casos de atletas que defendem publicamente a utilização da imaginação e prática mental com a finalidade de maximizar seus rendimentos no desporto que praticam. Coelho et al (2008) afirmam que a imaginação é a técnica mais utilizada para o treinamento mental, este processo consiste em imaginar a prática de um comportamento motor repetidas vezes de uma habilidade motora específica. Porém esta técnica não será eficaz se o atleta não souber quando e onde usá-la.

Na tabela 2 estão os dados descritivos dos escores de desempenho do fundamento recepção antes a após o período destinado a intervenção nos grupos controle e experimental. Os escores indicam os percentuais acerto dentro dos critérios determinados no protocolo do estudo. 
Tabela 2. Apresentação dos dados descritivos do desempenho do fundamento recepção das atletas dos grupos controle e experimental, nos momentos pré e pós intervenção.

\begin{tabular}{c|c|c|c|c}
\hline & \multicolumn{2}{|c|}{ Grupo Controle } & \multicolumn{2}{c}{ Grupo Experimental } \\
\cline { 2 - 5 } & Pré-intervenção & Pós-Intervenção & Pré-intervenção & Pós-Intervenção \\
\hline Mínimo & 65 & 50 & 45 & 55 \\
\hline Mediana & 80 & 75 & 57.5 & 70 \\
\hline Máximo & 95 & 90 & 70 & 80 \\
\hline Média & $\mathbf{8 0}$ & $\mathbf{7 4}$ & $\mathbf{5 7}$ & $\mathbf{6 8}$ \\
\hline Desvio Padrão & 7.6 & 13 & 7.5 & 9.2 \\
\hline
\end{tabular}

Destaques dados nos escores da tendência central de acordo com a normalidade dos dados.

A tabela evidencia que o grupo Controle apresentou uma redução de $8,1 \%$ da avaliação pré para a pós intervenção, em contrapartida o grupo Experimental apresentou um aumento de 19,3\% nessa mesma comparação, comprovando que houve melhora no rendimento do fundamento de recepção para atletas desse grupo. Tal informação torna-se mais relevante quando se traz a luz a informação de que as atletas que formavam a equipe sorteada como grupo Controle tinham de 1 a 2 anos a mais de prática no voleibol. Haja visto que o fato de ter menos tempo de prática no esporte poderia impor mais dificuldade à tarefa de mentalizar a técnica específica avaliada.

Em um estudo feito por Coelho et al (2008) com 46 jogadores de tênis de Campo do sexo masculino, com faixa etária média de 15,06 cujo a intervenção consistia em 5 minutos de vídeos, 5 minutos de relaxamento, 10 minutos de imaginação, mostrou que o grupo experimental demonstrou melhora em seu desempenho na situação saque sem precisão após as intervenções.

Além disso, em estudo recente feito por Fortes et al (2017) onde o objetivo foi analisar o efeito do treinamento mental sobre o desempenho de 35 jovens nadadores nos 100 metros e 200 metros livre, com idades entre 15 e 17 anos, em que o grupo experimental passou por 3 intervenções semanais durante 8 semanas com intervalos de 48 horas e duração de no máximo 10 minutos. O estudo concluiu que o treinamento mental foi eficiente na potencialização no desempenho dos atletas nas provas. Ainda, Castro e Santos (2007) analisaram em sua pesquisa a eficiência do treinamento mental na aprendizagem de reversão simples na ginastica olímpica de solo em atletas iniciantes na aprendizagem da técnica com idades de 9 a 13 anos. O grupo experimental teve o treinamento mental de aproximadamente 10 minutos em 16 sessões de 2 vezes na semana, e, o resultado extraído desse artigo mostrou que além de melhora técnica do grupo experimental, houve um aumento na qualidade da postura, na concentração e a diminuição de erros da execução do elemento reversão simples.

Terminada a apresentação descritiva dos escores da capacidade imaginativa e do desempenho no teste de recepção aplicado as participantes da pesquisa, se faz necessário verificar 
se as diferenças encontradas são suficientes para se comprovar de que o treinamento mental seria efetivo para melhoria do fundamento recepção. Para isso, é necessário se apresentar os valores de $\mathrm{p}$ referentes às análises inferenciais feitas intra e inter grupos e expostas na tabela 3.

Tabela 3. Teste T pareado feito para comparação dentro dos grupos (intra-grupo).

\section{Teste T pareado}

\begin{tabular}{c|c|c}
\hline \multirow{2}{*}{} & \multicolumn{2}{|c}{ Escore de Desempenho no teste de Recepção } \\
\cline { 2 - 3 } & Controle & Experimental \\
\cline { 2 - 3 } & Pré x Pós Intervenção & Pré x Pós Intervenção \\
\hline P valor & 0.1026 & 0.0011 \\
\hline P valor sumário & ns & $* *$ \\
\hline
\end{tabular}

O Teste T pareado comprova a evolução do grupo experimental, que no momento da pré intervenção, apresentava menor desempenho no fundamento recepção em relação ao grupo controle, porém ao final das intervenções no momento de pós intervenção houve uma grande melhora na quantidade de acertos na recepção dos saques.
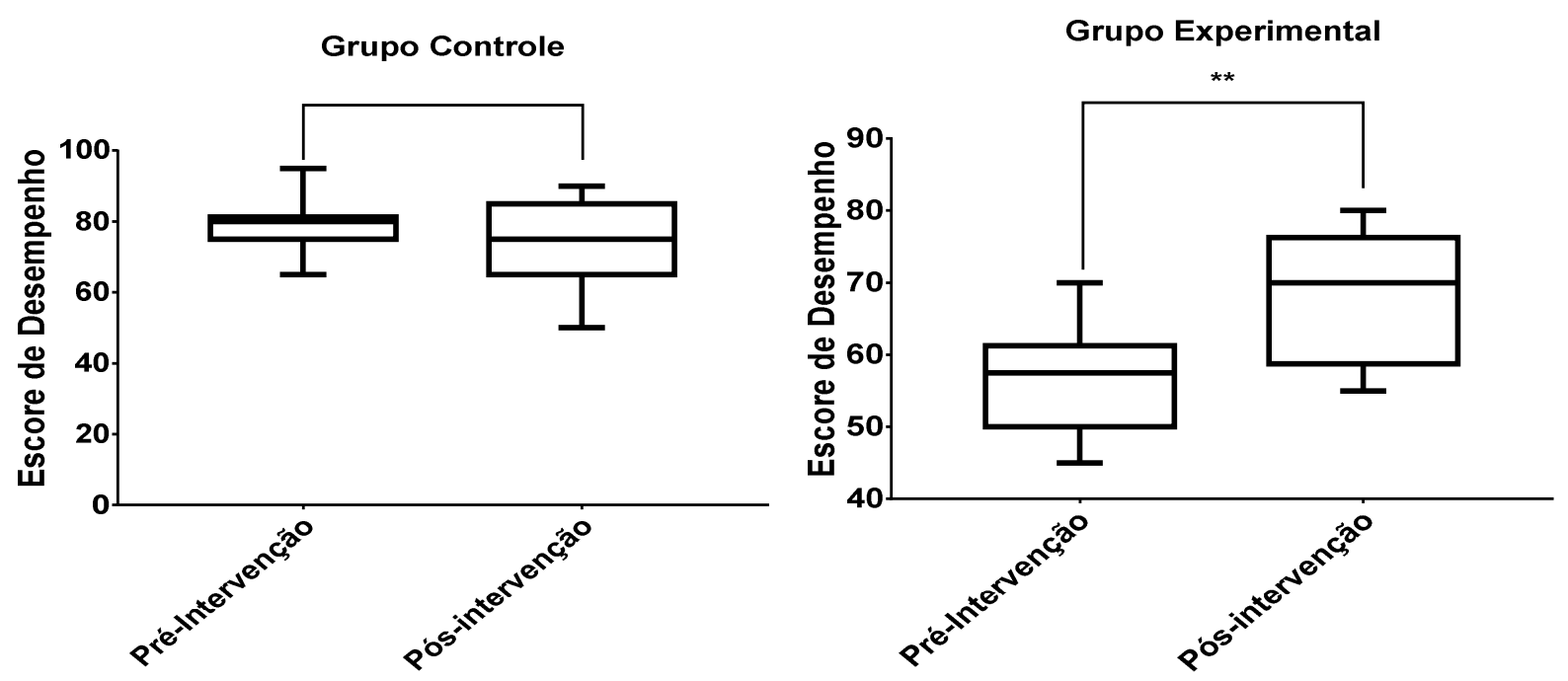

Figura 2. Comparações de desempenho intragrupos.

Para se finalizar as análises inferenciais, a tabela 4 traz os valores de $\mathrm{p}$ referentes as comparações intergrupos. 
Tabela 4. Teste T não-pareado feito para comparação entre os grupos (inter-grupo).

Teste T não-pareado

\begin{tabular}{c|c|c}
\hline \multirow{2}{*}{} & \multicolumn{2}{|c}{ Controle x Experimental } \\
\cline { 2 - 3 } & Pré-Intervenção & Pós-Intervenção \\
\hline P valor & $<0.0001$ & 0.2647 \\
\hline P valor sumário & $* * * *$ & Ns \\
\hline
\end{tabular}

O valor de p expresso na tabela 4 na comparação dos momentos pré-intervenção reforça que inicialmente os grupos possuíam qualidades técnicas diferentes, o grupo controle mostrou mais equilíbrio no escore de desempenho da recepção, provavelmente pelo fato de todas as atletas desse grupo possuírem maior tempo de prática na modalidade. Essa diferença entre os grupos não se manteve na avaliação pós-intervenção pois, a evolução promovida pela intervenção no grupo experimental fez com que na comparação após a intervenção, estatisticamente, a diferença entre os grupos desaparecesse, conforme representado na figura 3.

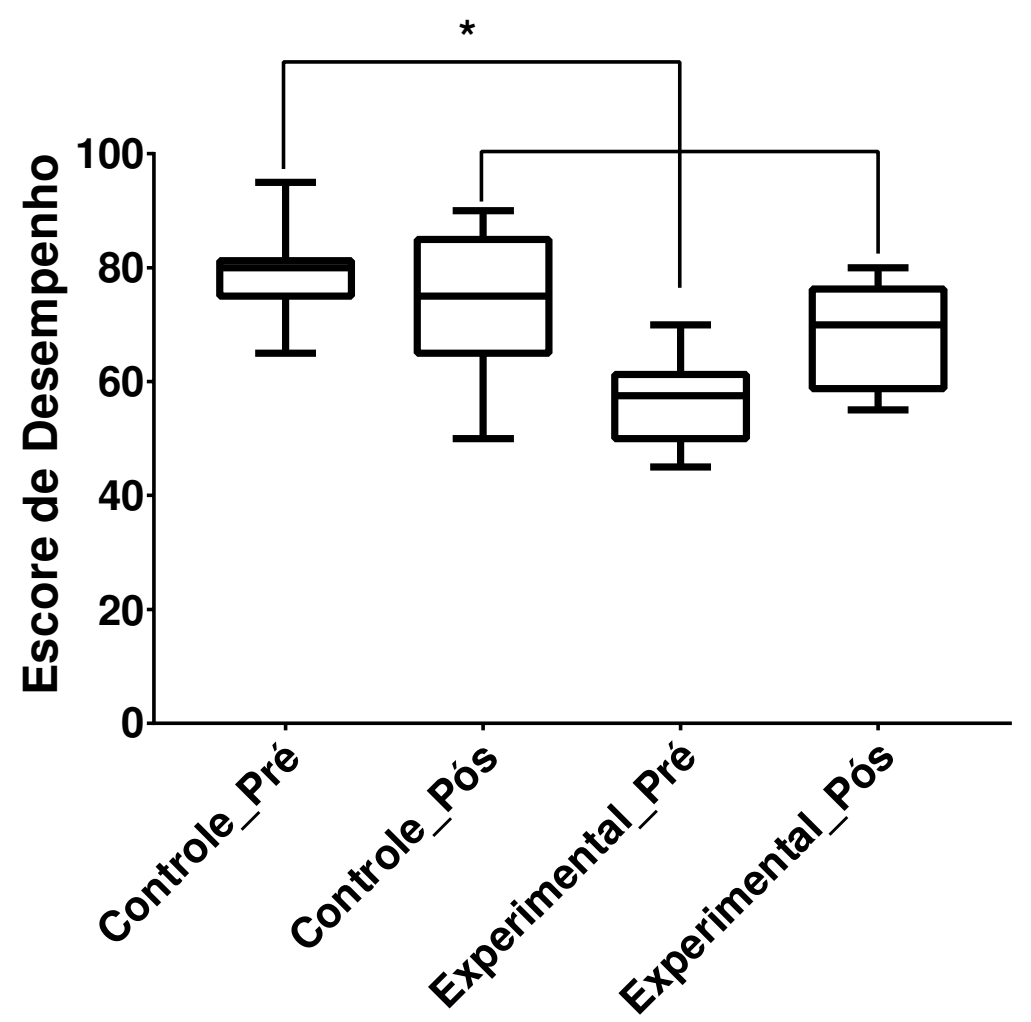

Figura 3. Figura ilustrativa da comparação de desempenho no fundamento recepção intergrupos.

* representa diferenças estatisticamente significativas. 


\section{CONSIDERAÇÕES FINAIS}

Os resultados apresentados constataram que mesmo comparando duas equipes que a priori demonstraram ser estatisticamente diferentes no que diz respeito ao desempenho no fundamento recepção de manchete, após o período de intervenção aplicado no grupo experimental o déficit existente passou a não existir. Apontando uma possível efetividade do treinamento mental na melhoria do fundamento recepção nas participantes do estudo.

Apesar da amostra desta pesquisa ser de atletas de voleibol amadoras, os resultados parecem corroborar com as evidências científicas a respeito da importância da aplicabilidade do treinamento mental nos desportos de alto rendimento, tal qual nas categorias de base. Os resultados também permitem se especular que apesar da menor experiencia do grupo experimental, a prática do treinamento mental ajudou a consolidar os engramas motores que normalmente se consolidariam por meio da prática específica do fundamento recepção. O que não ocorreu em ambos os grupos durante o período do estudo.

Por fim, é necessário se assumir que a diferença de experiencia existente entre os grupos do estudo é um fator limitante. Sendo assim, sugere-se que mais estudos possam verificar se a diferença no tempo de prática existente entre os grupos dessa pesquisa também não pode ser um viés que pode ter comprometido os resultados ora apresentados. Todavia, acredita-se que ao se replicar esses testes com outros grupos em outras modalidades, se possa ratificar os efeitos do treinamento mental e a importância da capacidade imaginativa em diferentes contextos esportivos.

\section{REFERÊNCIAS}

ALMEIDA, M. W. S. et al. Efeitos da imagética associado à música na melhora do arremesso de lance livre no basquetebol: comparativo entre dois grupos etários. Fitness \& performance journal, v. 7, n. 6, 2008.

BRANCO, L. P. et al. Efeitos De Um Protocolo De Treinamento Mental Associado À Estimulação Cerebral Proporcionando Potência Dos Membros Inferiores De Atletas De Voleibol. Perspectivas Online: Biológicas \& Saúde, vol. 7, nº. 24, 2017.

CASTRO, G. G.; SANTOS, F. C. P. Treinamento mental na aprendizagem do elemento reversão simples por crianças iniciantes na ginástica artística de solo. Movimentum Revista Digital de Educação Física, Ipatinga, vol.2, n.2, 11 p. ago/dez. 2007.

COELHO, R. W. et al. O efeito da imaginação no desempenho e na precisão do saque no tênis de campo. Revista Brasileira de Cineantropometria \& Desempenho Humano. v. 10, n. 2, p. 176$183,2008$.

FAGUNDES, F. M. et al. As interações motrizes do saque e da recepção e suas influências no voleibol: uma compreensão praxiológica. Motrivivência, v. 29, p. 225-242, 2017.

FILGUEIRAS A. et al. O uso da imagética mental como técnica para melhora do aproveitamento no futebol de campo: um estudo de caso. Coleção Pesquisa em Educação Física, vol.11, n.4, 2012 
FORTES, L. de S. et al. Efeito do treinamento mental no desempenho de jovens nadadores. Revista Brasileira de Ciência e Movimento, v. 25, n. 4, p. 23-31, 2017.

GOMES, T. B.; MARINHO, N. S.; BENDA, R. N. Imaginação e Treinamento Mental na Natação. REVISTA DE TRABALHOS ACADÊMICOS-UNIVERSO BELO HORIZONTE, v. 2, n. 1, 2016.

MENDES, P. A. D. Imagery: correlação entre o motor imagery e as habilidades e destrezas globais no futebol, nos gestos técnicos do passe, drible e remate em crianças de 12 e 13 anos. 2012. Dissertação de Mestrado, Universidade Castelo Branco : IPCB. Escola Superior de Educação. 89 f. 2012.

ORNELAS, C. S. et al. O EFEITO DA PRÁTICA DE MUSCULAÇÃO ASSOCIADA AO TREINAMENTO MENTAL E ESTIMULAÇÃO CEREBRAL NO DESENVOLVIMENTO DO BÍCEPS BRAQUIAL. Biológicas \& Saúde, v. 7, n. 23, 2017.

REZENDE, P. E. N.; SILVA, T. C.; SOUZA, R. F. EFEITO DO TREINAMENTO MENTAL NA CONCENTRAÇÃO PARA O ARREMESSO DE PESO. In: Congresso Internacional de Atividade Física, Nutrição e Saúde. 2016.

RIBAS, J. F. M; BALDICERA, M. C. R; ARAÚJO, P. A; TOGNI, E. Preparando Para o Ataque: Recepção. In: Ribas, J. F. M. (Org.) Praxiologia Motriz e Voleibol - Elementos para o Trabalho Pedagógico. (p. 69-80). Ijuí, Editora UNIJUÍ, 2014.

RODRIGUES, E. C. et al. Mental stimulation strategy affects postural control. Brazilian Journal of Psychiatry, v. 25, p. 33-35, 2003.

VIANA, M. F. Treino de imaginação e visualização mental. In: CRUZ, J. F. A. Manual de Psicologia do Desporto. Portugal: Lusografe-Braga. 1996. Vol 1, p. 627-648. 